\title{
New Methods in Materials Characterisation with Energy and Spatially Resolving X-ray Detectors
}

\author{
$\underline{\text { Henry J Kirkwood }}^{1 *}$, Martin J de Jonge² and Brian Abbey³ \\ 1. European XFEL GmbH, Schenefeld, 22869, Germany \\ 2. Australian Synchrotron, Clayton, Victoria, 3168, Australia \\ 3. Australian Research Council Centre of Excellence in Advanced Molecular Imaging, Department of \\ Chemistry and Physics, La Trobe Institute for Molecular Science, La Trobe University, Victoria \\ 3086, Australia \\ * Corresponding author, henry.kirkwood@xfel.eu
}

Using X-rays to map the elemental, chemical and structural properties of polycrystalline materials at the micro-scale typically requires multiple detectors or multiple instruments and hence the correlation of different results post-experiment. A new generation of X-ray detectors are currently emerging, which combine both energy and spatial resolution. These detectors are opening up a range of new opportunities for materials characterisation by combining multiple experiments into a single detector. The Maia [1], a pixelated energy-dispersive, area-sensitive, X-ray detector enables the simultaneous collection of X-ray fluorescence (XRF) and spatially-resolved X-ray diffraction (XRD) data. This class of detector is driving the development of novel types of materials science characterization experiments. Mapping elemental composition throughout the bulk of materials is a staple technique at most synchrotrons and is a key part of materials analysis. Similarly, determining the crystallographic orientation and residual elastic strain within a sample is crucial for understanding and predicting a materials' structural behaviour and response to external stresses. Here we highlight the capabilities of the Maia detector for simultaneously collecting XRF and XRD data from a polycrystalline sample (a Ni foil) and the methods developed to characterize the crystallographic structure, elemental composition and surface topography across the grain structure.

These experiments were performed at the X-ray fluorescence microprobe (XFM beamline) at the Australian Synchrotron [2] which is an advanced XRF instrument optimised for fast elemental and chemical mapping. The incident X-ray beam at XFM passes through the centre of the detector to the focal plane, located $10 \mathrm{~mm}$ downstream of the Maia. The close detection geometry means that the Maia subtends a large solid angle and is able to collect complete spectral information over the full energy range of the incident energy and fluorescence spectra. By scanning the incident energy over an appropriate range, the X-ray absorption fine structure of the material can also be measured.

Although the array of 384 detector pixels in the Maia allows for only limited spatial resolution, the additional energy resolution information is incorporated into the crystallographic analysis. The Maia also offers many benefits compared to other XRF detectors. Collecting XRF information over the large solid angle covered by the Maia permits more XRF signal to be collected in less time, leading to much reduced dwell times and hence faster sample scanning as well as the collection of elastic diffraction data. We demonstrate that the Maia resolution is sufficient to determine crystallographic orientation uniquely provided at least 4 Bragg reflections are measured. These reflections are then used to estimate the 
residual elastic strain [3]. We describe how small changes in the spatial distribution of XRF can be attributed to changes in the sample surface normal and present a method for determining the relative surface relief from the XRF distribution [4], which we find compares well to measurements made with a scanning electron microscope, shown in figure 1.

The diffraction peak profile analysis for Bragg reflections measured from a single grain are also presented and discussed. We observe features that indicate the presence of either crystallographic twins or active slip planes. In this case the number of measured Bragg peaks ( 2 in this case) from the parent grain was not sufficient to determine the crystallographic orientation uniquely. Measuring additional Bragg peaks would allow the orientation to be determined and hence the source of the crystallographic features. Further analysis of this grain by electron backscatter diffraction (EBSD) confirmed that there were indeed slip planes present, formed during the high temperature annealing of the polycrystalline foil shown in figure 1.

Methods have been developed for combining spectral and spatial information from proof-of-principal experiments using the Maia for materials characterisation. There are still significant spatial resolution challenges which may be overcome with future generations of X-ray detectors.

\section{References:}

[1] R Kirkham et al, In AIP Conf. Proc., 1234(1), 240-243

[2] Paterson et al, In AIP Conf. Proc., 1365(1), 219-222

[3] Kirkwood et al, Acta Materialia, 144 (2018), 1-10

[4] Kirkwood et al, Powder Diffraction, 9 (2017), 1-6

(a)

(b)
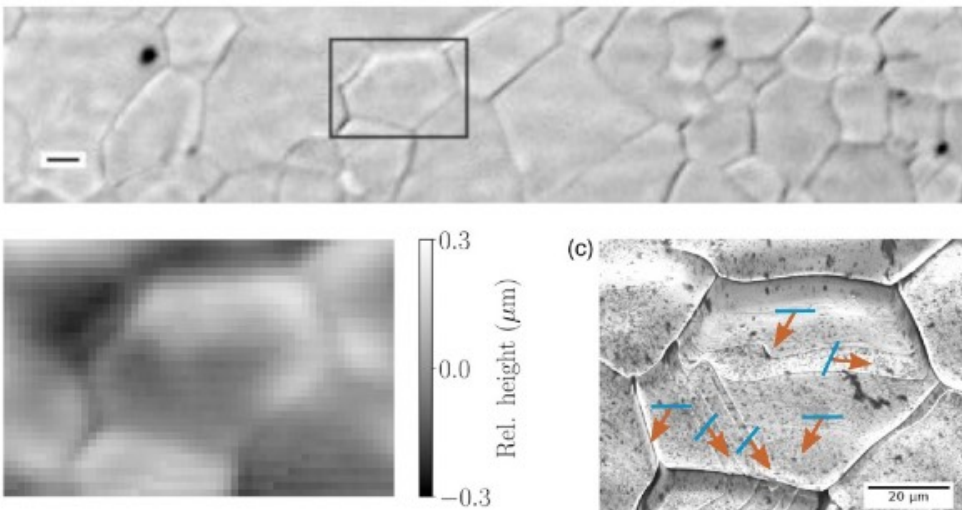

(c)

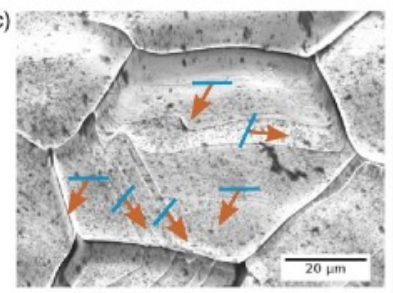

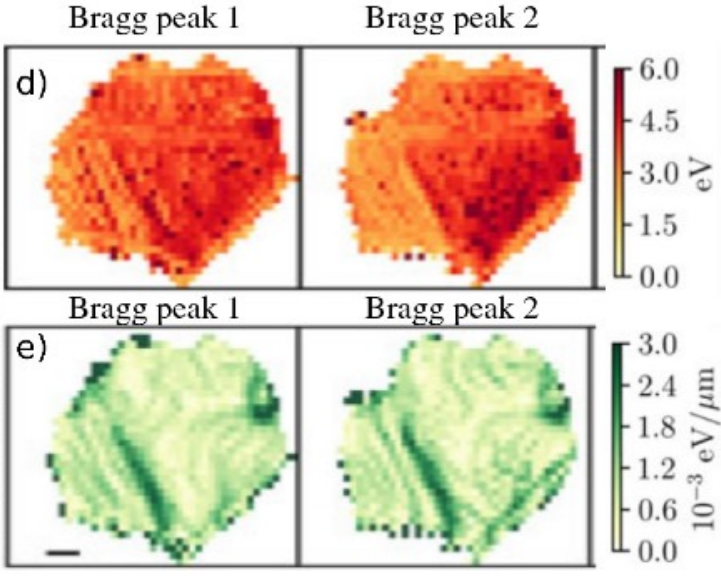

Figure 1. Simultaneous XRD and XRF measurements capitalising on the high energy resolution and modest spatial resolution of the Maia detector. a) Ni XRF intensity across a polycrystalline Ni foil sample; scale bar $20 \mu \mathrm{m}$. b) Surface relief map inferred from non-uniformities in Ni fluorescence distribution. c) The topography as visualised with a SEM, in good agreement with the x-ray data. Slip planes and directions as determined with EBSD overlaid. Analysis of the x-ray diffraction maps acquired at many energies enables determination of d) the Bragg peak FWHM and e) magnitude of the spatial gradient of the Bragg peak energy, clearly visualising the slip plane associated features. 